\title{
Isolated mesopic rod and cone electroretinograms realized with a four-primary method
}

\author{
Dingcai Cao, \\ Department of Surgery, Section of Ophthalmology and Visual Science, The University of Chicago, \\ 940 E. 57th Street, Chicago, IL 60637, USA
}

Joel Pokorny, and

Visual Science Laboratories, The University of Chicago, 940 East 57th Street, Chicago, IL 60637, USA

Michael A. Grassi

Department of Surgery, Section of Ophthalmology and Visual Science, The University of Chicago, 940 E. 57th Street, Chicago, IL 60637, USA

\begin{abstract}
The purpose of this study was to evaluate the feasibility of measuring rod and cone electroretinograms (ERGs) at a single mesopic adaptation level. To accomplish this, a fourprimary photostimulator was implemented using a commercially available ERG system (Diagnosys ColorDome) to generate three types of stimuli that temporally modulated rods alone, cones alone, and rods and cones simultaneously. For each stimulus type, ERGs were recorded as a function of temporal frequency $(2,4,8$, or $16 \mathrm{~Hz})$ and mesopic light levels $(0.02,0.16$, or $1.26 \mathrm{~cd} /$ $\mathrm{m}^{2}$ ) in normal observers and patients with retinitis pigmentosa (RP) or cone-rod degeneration. The normal observers ERG waveforms showed a clear periodic pattern, mirroring the sinusoidal stimuli. At all light levels, rod responses were always higher than cone responses for temporal frequencies between 2 and $8 \mathrm{~Hz}$, suggesting that rods dominated the responses. Cone responses were minimal at the lowest light level and increased with increases in light level. The amplitude of the response to the combined stimuli was intermediate between that of the isolated cone and the isolated rod stimuli for all light levels. Good receptoral isolation was confirmed by the results showing (1) minimal or no rod ERGs but recordable cone ERGs in the patients and (2) high correlation between the ERG amplitudes obtained from the four-primary method and those from the ISCEV standard clinical protocol in normal observers.
\end{abstract}

\section{Keywords}

Mesopic; Rod; Cone; ERGs; Four-primary; Rod-cone interaction

\section{Introduction}

Full-field electroretinography is a non-invasive ocular electrophysiological test that is widely used for retinal disease diagnosis and evaluation. It is known that certain retinal

\footnotetext{
(C) Springer-Verlag 2011

Correspondence to: Dingcai Cao.

d-cao@uchicago.edu .

Present Address: M. A. Grassi Department of Ophthalmology and Visual Science, Lions of Illinois Eye Research Institute, University of Illinois at Chicago, 1905 W. Taylor Street, \#155, Chicago, IL 60612-7244, USA
} 
disorders may selectively affect rods (e.g., retinitis pigmentosa) or cones (e.g., cone dystrophies), resulting in a decrease in either the rod- or the cone-driven ERGs. Therefore, for clinical evaluation, it is necessary to measure rod and cone electroretinograms (ERGs) separately. The pioneering work by Berson and Gouras provided the foundation for developing a clinical ERG protocol to probe rod and cone function (e. g., [1, 2]).

A variety of strategies have been used to isolate rod and cone ERGs, such as the manipulation of adaptation and/or temporal parameters [3]. The adaptation strategy involves changing luminance level and adaptation status (dark vs. light adaptations). Rod ERGs are measured at a scotopic light level following 20-30 min of dark adaptation, and cone ERGs are measured at a much higher photopic light level following light adaptation. This strategy takes advantage of the fact that the rods and cones operate best at different light adaptation levels and that rods become saturated at high light levels. This is the procedure that has been adopted as the recommended standard protocol for rod and cone ERGs testing by the International Society for Clinical Electrophysiology of Vision (ISCEV) [4]. Additionally, manipulation of the temporal parameters takes advantage of the differences in the temporal response characteristics of the rod and cone systems. The rod ERGs has a lower flicker fusion frequency than the cone ERGs [5]; therefore, a measurable 30-Hz flicker ERGs is considered to be cone driven and is recommended by the ISCEV for cone ERG measurement [4].

In this study, we used a unique approach for measuring isolated rod and cone ERGs. This approach allows the measurements of rod and cone ERGs at mesopic light levels where rods and cones are both responsive. We used a four-primary method that controlled the excitations of rods and the three types of cones independently with a silent substitution method [6, 7]. Following the colorimetric principle, three primaries are required to "silent" two photoreceptor types while stimulating one photoreceptor type only, such as generating $L$-cone modulation while keeping $M$ - and $S$-cone excitations constant on CRT. Since at mesopic light levels, all of four photoreceptor types (rods, $L-, M$ - and $S$-cones) are active, it is necessary to use four primaries to isolate one photoreceptor excitation while keeping the other three constant. The four-primary method was implemented with a commercially available electrophysiology system that included a Ganzfeld stimulator illuminated by 4 light-emitting diodes (LEDs). We applied the four-primary principles to generate stimuli that modulated rod excitations alone, cone excitations alone, or combined rod and cone excitations, and measured ERGs with these stimuli. The Ganzfeld presents a full-field stimulus that covers a major portion of the retina. The spectral sensitivity functions for the rods and cones derived from the $10^{\circ} \mathrm{CIE}$ color matching functions may not be applicable for a full-field stimulus due to macular pigment [8] or photoreceptor optical density [9], leading to an incomplete isolation of rod and cone excitations. To test this, we measured rod or cone ERGs using the four-primary method in patients with retinitis pigmentosa (RP) or cone-rod degeneration, who, based on the ISCEV standard clinical protocol [4], had minimal measurable rod ERGs and slightly decreased cone ERGs. If the isolation of rod and cone excitations was incomplete, we would expect some measurable ERGs with the isolated rod stimuli in the patients. Further, we correlated rod or cone ERG amplitudes obtained from the method with those from the ISCEV standard protocol. Our results indicate that the new method can effectively separate rod and cone ERGs at mesopic light levels. This finding is corroborated by ERG measurements for patients with RP or cone-rod degeneration, and a high correlation between ERG amplitudes measured by this method and those by the ISCEV standard protocol. Under the same testing conditions, the patients did not have measurable ERGs with the isolated rod stimuli but had measurable ERGs with the isolated cone stimuli. The cone ERG amplitudes from the patients were smaller than those from normal observers, consistent with the results from ISCEV standard ERG protocol. 
The second purpose of this study was to investigate rod-cone interactions in ERGs. Clinical ERGs testing typically uses pulsed (flashed) stimuli to evaluate receptoral and postreceptoral contributions to ERGs. To investigate the rod-cone interaction, periodic (flickering) stimuli were used because phase information is available and time-averaged state of adaptation can be held constant [10]. Psychophysically, rod-cone interactions have been investigated for many aspects of visual function, including increment detection, chromatic discrimination, color perception, temporal processing, and spatial processing [11]. In particular, rod and cone signals in flicker detection can be described by a vector sum model $[12,13]$. Primate ganglion cell recordings indicate that rod and cone responses are combined linearly prior to the saturation site [14]. However, little is known about rod-cone interactions in full-field ERGs [15]. Our results indicated that rod and cone ERGs are combined linearly following a vector sum model in normal observers, consistent with the findings of Kremers and Scholl [16].

\section{Methods}

\section{Apparatus and calibration}

Full-field ERG measurements were obtained using an Espion ${ }^{2}$ electrophysiology system (Diagnosys LLC, Lowell, MA, USA) that includes a ColorDome Ganzfeld. The ColorDome Ganzfeld contains three rings of LEDs, a "bright ring," a "dim ring," and a "low dim ring" to achieve a large dynamic range. For this study, we only used the "dim ring" that was sufficient to produce light levels within mesopic range. The "dim ring" had 4 LEDs with dominant wavelengths as $470 \mathrm{~nm}$ ("blue"), $524 \mathrm{~nm}$ ("green”), $588 \mathrm{~nm}$ ("amber"), and 636 $\mathrm{nm}$ ("red") $\mathrm{nm}$. The ColorDome Ganzfeld was programmed to serve as a four-primary photostimulator that could control rod and cone stimulations independently. The theoretical basis for achieving independent control of the activities of four types of photoreceptors ( $S$ cones, $M$-cones, $L$-cones, and rods) in the human retina is silent substitution [6, 17]. The cone excitations were computed based on the Smith-Pokorny cone fundamentals for the CIE $196410^{\circ}$ Standard Observer [6]. The cone chromaticities were described in a relative cone troland space, which plots $S /(L+M)$ versus $L /(L+M)$ [18]. For an equal-energy-spectrum (EES) light, the $L /(L+M)$ value is 0.667 and the $S /(L+M)$ value is 1.0. The cone luminance is the sum of the $L$ and $M$ cone excitations and is specified in photopic Trolands. Rod excitation was computed based on the scotopic luminous efficiency function, $V^{\prime}(\lambda)$, with normalization such that 1 photopic Troland of EES light defines rod excitation of 1 rod Troland [6]. Using Maxwellian-view four-primary photostimulators [7, 19], we have conducted psychophysical and physiological studies that have not only yielded results consistent with literature but also provided new insights of rod and cone functions, demonstrating the viability of the four-primary method in investigating mesopic rod and cone functions (e.g., [14, 20-23]).

Since the built-in calibration provided by Diagnosys was based on the CIE $19312^{\circ}$ standard observer, we calibrated the light outputs from the ColorDome LEDs so that we could specify stimuli in the CIE $196410^{\circ}$ colorimetric system. It is necessary to establish the spectral distribution and input-output relation of each of the LEDs. The spectral distribution of each LED was measured with a PhotoResearch PR-650 spectroradiometer. The CIE $10^{\circ}$ luminance of each LED at its maximum was calculated from the spectral measurements. For linearization, the light output of each LED as a function of the digital voltage level was measured using a PIN silicon diode combined with a current amplifier and precision voltmeter. Each LED output was linearized using 3 linear equations for the ranges of $0.1-$ $1.0,0.01-0.1$, and $0.001-0.01$ of the maximum LED output [24]. 


\section{Stimuli}

We generated three types of sinusoidal stimuli in the ColorDome Ganzfeld at each light level: (1) isolated rod stimuli (only rod excitation was modulated while maintaining constant $L-, M$ - and $S$-cone excitations, that is, $L-, M$ - and $S$-cone excitations had zero contrast), (2) isolated cone stimuli (only cone luminance, $L+M$, was modulated while rod and $S$-cone excitations were kept constant, that is, $L$ - and $M$-cone excitations were modulated in phase at the same contrast while rod and $S$-cone excitations had zero contrast), and (3) combined rod and cone stimuli (both rod and cone luminance signals were modulated in phase, that is, $L$ and $M$-cone and rods were modulated while $S$-cone excitation had zero contrast). To achieve a large range of contrast for rod or cone modulation, the time-averaged chromaticity for all of the stimulus types was $L /(L+M)=0.75$ and $S /(L+M)=0.20$ (the field appeared a desaturated orange) in a relative cone troland space [18], corresponding to $\mathrm{CIE} 10^{\circ}$ coordinates of $x=0.5342$ and $y=0.3883$. The rod and/or cone excitations ( $L$ - and $M$-cones only) were modulated sinusoidally with a Michelson contrast of $30 \%$ and a temporal frequency of $2,4,8$, or $16 \mathrm{~Hz}$. The time-averaged photopic luminances were $0.02,0.16$, and $1.26 \mathrm{~cd} / \mathrm{m}^{2}$. For an $8-\mathrm{mm}$ pupil, these lights gave corresponding retinal illuminances of 1,8 , and 63 photopic $\mathrm{Td}$, or approximately $0.49,3.9$, and 31 effective photopic $\mathrm{Td}$ when the Stiles-Crawford effect is taken into account [25]. The changes in luminance were achieved by adding calibrated 0.9 and $1.8 \log$ unit neutral density filters to cover the ColorDome for the 0.16 and $0.02 \mathrm{~cd} / \mathrm{m}^{2}$ light levels. For the time-averaged chromaticity for all of the stimulus types, the scotopic-to-photopic luminance ratio was 1.17 ; therefore, the scotopic luminances were $0.023,0.19$, and 1.48 scotopic $\mathrm{cd} / \mathrm{m}^{2}$, respectively.

\section{Observers}

Thirteen healthy observers ( 4 men and 9 women) with normal color vision (assessed by the Nagel anomaloscope and FM-100 hue test) with a best-corrected visual acuity of at least 20/20 were tested. The mean age of normal observers was 29 years (range, 18-62 years). Six patients ( 3 women and 3 men), who were diagnosed by the author M.A.G., were recruited from the University of Chicago Hospitals Retina Clinic. The mean age of the patients was 44 years (range, 27-65 years). Five of the patients had RP, while one had cone-rod degeneration. The genetic mutations were known for some of the patients from chart review. One RP patient had mutation in ORF15 of the RPGR gene (X-linked RP, man, age 28 years), and the second RP patient had the E3731Q and Q4662E variants in the USH2A gene (simplex RP, recessive, man, age 57 years). The remaining $3 \mathrm{RP}$ patients were diagnosed as autosomal dominant RP (man, age 65 years), autosomal recessive RP (woman, age 47 years), and simplex RP (woman, age 38 years). Another patient (woman, age 27 years) had cone rod degeneration, which has different rates of rod and cone function loss from other forms of RP [26]. We included this patient because her measured rod and cone ERGs with the ISCEV standard protocol were similar to those of the RP patients.

All of the RP patients and 12 out of the 13 normal observers underwent the ISCEV standard protocol for rod ERGs, mixed rod and cone ERGs, and cone ERG measurements [4]. The measurements were taken with the Xenon light source in the ColorDome stimulator using the built-in ISCEV standard protocol in the Diagnosys Espion ${ }^{2}$ electrophysiology system. The protocols were approved by the University of Chicago Institutional Review Board and adhered to the tenets of the Declaration of Helsinki.

\section{Observer calibration}

To achieve good isolation of rod and cone stimuli at mesopic light levels, it is necessary to consider individual differences in pre-receptoral filtering (principally lens transmittance). For the Maxwellian-view four-primary photostimulator [7], a color-matching technique was used to estimate individual pre-receptoral filtering compared with the standard observer 
[20]. To estimate the individual differences in pre-receptoral filtering for the ERG measurements, we used b-wave amplitudes of scotopic ERGs measured with a single flash (4 ms) generated by the "blue," "green," "amber," or "red" LED alone in the ColorDome stimulator at 0.005 or $0.05 \mathrm{scotopic} \mathrm{cd} / \mathrm{m}^{2}$, using the built-in calibration values of each LED in the Diagnosys Espion ${ }^{2}$ electrophysiology system. The scotopic ERGs were repeated 6 times for each LED for each normal observer. The averaged b-wave amplitude from the "blue," "amber," and "red" LED was divided by the averaged amplitude from the "green" LED to calculate the relative amplitude ratios at each light level. The within-observer variation in the amplitude ratios at both light levels $\left(0.005\right.$ and $\left.0.05 \mathrm{~cd} / \mathrm{m}^{2}\right)$ was very small; therefore, we averaged the amplitude ratios obtained from the two light levels. These amplitude ratios were used to compensate for the difference between standard observer and each of our observers in the generation of isolated rod or cone stimuli. In patients, it was impossible to measure scotopic ERGs to assess individual differences in pre-receptoral filtering, and we used the averaged correction factor from the first five normal observers for patient testing.

\section{Procedure: ERG recording}

The eyes were dilated with $1 \%$ tropicamide drops and dark adapted for 30 min before ERG measurement. ERGs were recorded binocularly with bandwidths of 0.3 and $300 \mathrm{~Hz}$ at a $1,000-\mathrm{Hz}$ sampling rate using DTL Plus corneal electrodes, which were referred to ear clip electrodes and a wrist electrode ground. Head position was maintained using a chin rest in front of the ColorDome stimulator.

Each session included three light levels: first, $0.02 \mathrm{~cd} / \mathrm{m}^{2}$; then, $0.16 \mathrm{~cd} / \mathrm{m}^{2}$; and finally, 1.26 $\mathrm{cd} / \mathrm{m}^{2}$. The observers adapted to a light level for $2 \mathrm{~min}$ before recording. At each light level, 12 conditions were tested, with each condition consisting of one type of stimulus (isolated rod, isolated cone, or combined stimuli) at one temporal frequency $(2,4,8$, or $16 \mathrm{~Hz})$. Data were collected over 2,000-ms period for a trial or a sweep with a 500-ms interval between trials. Individual trials that included an eye movement or blink artifact (i.e., maximum amplitude $\geq 200 \mu \mathrm{V}$ ) were removed automatically by the Diagnosys Espion ${ }^{2}$ electrophysiology system or manually by the ERG technician during the recordings. Three sweeps were recorded for each condition. One session lasted approximately $45 \mathrm{~min}$.

Five normal observers were tested for three or more sessions on different days. The other 8 normal observers and all 6 patients were tested for one or two sessions on the same day. Observers were given sufficient rest between conditions to avoid fatigue. One RP patient was not tested at $1.26 \mathrm{~cd} / \mathrm{m}^{2}$ because he could not tolerate the bright light. Another RP patient was not tested at the $16-\mathrm{Hz}$ condition because of very weak responses at $8 \mathrm{~Hz}$.

\section{Data analysis}

For all stimulus conditions, the ERG responses from the two eyes of each observer were similar and the data from the two eyes were averaged. The averaged waveform for each condition at a light level was subjected to a discrete Fourier transformation to extract the amplitude and phase of the first harmonic. Noise was estimated based on the amplitudes of the neighboring frequency $1 \mathrm{~Hz}$ above a test frequency $(2,4,8$, and $16 \mathrm{~Hz})$, an approach similar to the one used by Murray et al. [27]. For instance, for the test frequency of $4 \mathrm{~Hz}$, the amplitude at $5 \mathrm{~Hz}$ was used for noise estimation.

The difference in the extracted amplitude and noise amplitude for each condition was computed for each observer. If the amplitude was smaller than the noise level for a condition, the amplitude for that condition was set as zero for further statistical analysis. Then, the amplitudes with noise removed were compared between normal observers and 
patients for each stimulus type using a three-way repeated measures ANOVA, which included the main effects of disease diagnosis (normal vs. patient), frequency $(2,4,8$, and $16 \mathrm{~Hz})$, and light level $\left(0.02,0.16\right.$, and $\left.1.26 \mathrm{~cd} / \mathrm{m}^{2}\right)$, as well as two-way and three-way interactions between these factors.

We compared rod and cone ERGs obtained from the four-primary method and the ISCEV protocol. First, for each stimulus type (isolated rod stimuli, isolated cone stimuli, or combined rod and cone stimuli), we found each observer's maximal ERG amplitude across all temporal frequencies and light levels measured by the four-primary method. The resulting maximal amplitudes were correlated with the ERG amplitudes from the ISCEV standard protocol using linear regression analyses.

\section{Results}

\section{Rod and cone ERGs in normal observers and patients measured by the ISCEV standard protocol}

Compared with the normal observers, the patients showed much reduced rod ERGs [rod ERG b-wave amplitude (mean $\pm \mathrm{SD}$ ): $38.2 \pm 17.9 \mu \mathrm{V}$ in patients vs. $162.4 \pm 52.1 \mu \mathrm{V}$ in normals, $P<0.001]$. These patients had significantly reduced combined ERGs or cone ERGs compared with normal observers [standard combined ERG b-wave amplitude: $52.8 \pm$ $38.5 \mu \mathrm{V}$ in patients versus $387.1 \pm 123.5 \mu \mathrm{V}$ in normals, $P<0.001$; single-flash cone ERG b-wave: $17.7 \pm 10.0 \mu \mathrm{V}$ in patients vs. $99.5 \pm 27.6 \mu \mathrm{V}$ in normals, $P<0.001 ; 30-\mathrm{Hz}$ flicker cone ERG amplitude (peak-trough): $12.9 \pm 9.0 \mu \mathrm{V}$ in patients vs. $100.3 \pm 24.0 \mu \mathrm{V}$ in normals, $P<0.001]$.

\section{Rod and cone ERGs in normal observers and patients measured by the four-primary method}

Figure 1 shows the averaged waveforms of the measurements for one normal observer ( $Y S$, woman, 30 years old) on five different days of both eyes ( 30 sweeps in total for each condition were used for averaging, 15 for the left eye, and 15 for the right eye) with the isolated rod stimuli (left column), isolated cone stimuli (middle column), or the combined stimuli (right column). The higher frequency components $(>30 \mathrm{~Hz})$ in each waveform were digitally filtered out. Each panel shows the ERG waveforms with one stimulus type and one frequency at all three light levels (top trace for $1.26 \mathrm{~cd} / \mathrm{m}^{2}$, middle trace for $0.16 \mathrm{~cd} / \mathrm{m}^{2}$, and the bottom trace for $0.02 \mathrm{~cd} / \mathrm{m}^{2}$ ). For reference, the sinusoidal stimulus is plotted in the bottom in each panel. The ERG waveforms demonstrate clear periodic patterns that related to the sinusoidal stimuli.

The amplitudes and the phases of the first harmonics of waveforms in Fig. 1 from observer $Y S$ are shown in Fig. 2 (left column for amplitude and right column for phase). The amplitudes were all greater than noise estimates (dash lines) except for the higher frequencies $(8$ or $16 \mathrm{~Hz})$ at the lower light levels $\left(0.02\right.$ or $0.16 \mathrm{~cd} / \mathrm{m}^{2}$ ). At $0.02 \mathrm{~cd} / \mathrm{m}^{2}$ (top row in Fig. 2), the amplitude and phase with the isolated rod stimuli and the combined stimuli were almost identical, while the amplitude of ERGs with the isolated cone stimuli was much smaller than for the other two types of stimuli. At higher light levels (middle and bottom rows, Fig. 2), the amplitude with the isolated rod stimuli was the highest, and amplitude with the isolated cone stimuli was the lowest. Further, the phase with the isolated rod stimuli was similar to that with the combined stimuli, indicating that rod contributions to the ERG signals still dominated at these light levels. The rod phase had a difference of $\sim 200^{\circ}$ compared with the cone phase. The phases of the ERG responses with different stimulus types decreased linearly with temporal frequency (the dashed lines are linear 
regression fits), but the slopes were almost identical (the slopes between the rod and cone stimuli differed only $0.65^{\circ} / \mathrm{Hz}$ at $1.26 \mathrm{~cd} / \mathrm{m}^{2}$ and $0.97^{\circ} / \mathrm{Hz}$ at $0.16 \mathrm{~cd} / \mathrm{m}^{2}$ ).

Figure 3 shows the averaged amplitudes from all of the normal observers under different conditions, along with the averaged results from the patients. In the patients, the rod ERGs had small amplitudes ( $<3 \mu \mathrm{V}$, close to the noise level) at all frequencies and light levels. In normal observers, the rod ERGs had reliable amplitudes at $\leq 8 \mathrm{~Hz}$, which were significantly higher than those in patients [disease $\times$ frequency: $F(3,200)=26.16, P<0.001$; disease $\times$ light level: $F(2,200)=9.68, P<0.001$; main effect of disease: $F(1,200)=214.02, P<$ $0.001]$. With the isolated cone stimuli or combined stimuli, the patients showed weak ERG responses but reliably higher than the noise level, especially with the low frequency $(\leq 4 \mathrm{~Hz})$ and higher light level $\left(\geq 0.16 \mathrm{~cd} / \mathrm{m}^{2}\right)$ stimuli.

Normal observers had significantly higher cone ERG amplitudes than the patients [disease $x$ frequency: $F(3,200)=1.60, P=0.19$; disease $\times$ light level: $F(2,200)=6.50, P=0.002$; main effect of disease: $F(1,200)=21.44, P<0.001$ ]; normal observers also had significantly higher combined ERG amplitudes than the patients [disease $\times$ frequency: $F(3,200)=13.02$, $P<0.001$; disease $\times$ light level: $F(2,200)=1.92, P=0.15$; main effect of disease: $F(1,200)$ $=138.3, P<0.001]$.

\section{Correlations between ERG amplitudes measured by the ISCEV standard protocol and the four-primary method}

Figure 4 shows the correlation between the rod or cone ERG amplitudes measured by the four-primary method and the ISCEV standard protocol. In normal observers, the amplitudes of the rod ERGs (Fig. 4a), combined rod and cone ERGs (Fig. 4b) and cone ERGs (Fig. 4c) measured by the four-primary method were significantly correlated with the amplitudes of the ISCEV rod ERGs, 30-Hz cone ERGs and combined rod and cone ERGs (Pearson correlations $r$ between 0.63 and $0.82, P$ 's $\leq 0.028$ ). The amplitude of the cone ERGs had a non-significant correlation with that of the ISCEV single-flash cone ERGs (Pearson correlation $r=0.44, P=0.149$, Fig. 4d). In patients, the correlations for rod ERG amplitudes and cone ERGs were nearly significant $(r=0.79, P=0.064$ for rod ERGs, Fig. $4 \mathrm{a} ; r=0.75$, $P=0.084$ for cone ERGs), Fig. 4c), indicating that our approach was sensitive to detect small differences even when the overall amplitudes were small. Finally, the fits from linear regression models without intercepts can describe the data well in both normal observers and patients (see the dashed lines in Fig. $4, R^{2}$ between 0.91 and 0.97 for normal observers and 0.68 and 0.92 for the patients), suggesting that the measured amplitudes from the fourprimary method were nearly proportional to those from the ISCEV standard protocol.

\section{Combination of rod and cone ERGs}

To evaluate how rod and cone ERGs combine in normal observers, a vector sum model was used to predict the ERG amplitude and phase with the combined stimuli based on the ERGs with the isolated rod and cone stimuli. The ERGs to the isolated rod or cone stimuli with a temporal frequency, $f$, was described as a sinusoidal function with a rod or cone ERG phase $\phi_{\text {rod }}$ or $\phi_{\text {cone }}:$

$$
\mathrm{ERG}_{\mathrm{rod}}=A_{\text {rod }} \sin \left(2 \pi f t+\phi_{\text {rod }}\right)
$$

$$
\mathrm{ERG}_{\text {cone }}=A_{\text {cone }} \sin \left(2 \pi f t+\phi_{\text {cone }}\right)
$$


The ERGs with the combined stimuli were predicted by a vector sum of the ERGs with the isolated rod and cone stimuli:

$$
\mathrm{ERG}_{\text {combined }}=A_{\text {rod }} \sin \left(2 \pi f t+\phi_{\text {rod }}\right)+A_{\text {cone }} \sin \left(2 \pi f t+\phi_{\text {cone }}\right) .
$$

For Eq. 3, noise was not subtracted from the ERG amplitudes ( $A_{\text {rod }}$ and $\left.A_{\text {cone }}\right)$. We did not analyze the data obtained at 8 and $16 \mathrm{~Hz}$, because for these conditions, responses were small at all light levels and phase estimation was not reliable. Figure 5 shows the predicted amplitudes and phases based on the vector sum model versus the measured values from the 5 normal observers tested at least for 3 sessions; these data were chosen for analysis because they provided the most reliable phase information. Each point in each panel represents the value for one frequency from one observer. The dashed lines represent correspondence of the predicted and measured values. Clearly, the vector sum model described the amplitudes and phases with the combined stimuli well.

\section{Discussion}

The ISCEV standard protocol for full-field ERG measurements achieves isolation of rod and cone ERGs with the manipulation of adaptation and temporal parameters: Rod ERGs are measured using a dim white flash following dark adaptation for at least 20 min prior to recording, while cone ERG are measured using a single bright flash or a $30-\mathrm{Hz}$ flickering stimulus following a 10-min light adaptation to suppress rod activity [4]. Using the fourprimary method, we were also able to isolate rod and cone ERGs in normal observers, based on several lines of evidences. First, the fact that the combined stimuli produced amplitudes intermediate between the rod and cone amplitudes (see Fig. 2) indicates two separate photoreceptor classes that contribute to the ERGs. If a single photoreceptor class mediated both the putative rod and cone ERGs, the response amplitude would be the algebraic sum rather than the vector sum of the amplitudes. Second, the patients did not have recordable rod ERGs at mesopic light levels but had measurable cone ERGs. Further, the cone ERG amplitudes from patients were similar to those from normal observers at $0.02 \mathrm{~cd} / \mathrm{m}^{2}$ and slightly smaller than normal observers at higher light levels at 0.16 and $1.26 \mathrm{~cd} / \mathrm{m}^{2}$ (see Fig. 3 ). Third, the measured amplitudes for both the normal observers and the patients correlated well with the ISCEV counterparts (see Fig. 4). Finally, our analysis of the lens aging effect on photoreceptor isolation indicates that even there is some isolation error, the impact of isolation error is minimal for ERG responses. Using a mean luminance of $66 \mathrm{~cd} / \mathrm{m}^{2}(1.8 \mathrm{log}$ unit higher than the highest light level we used in our study $1.26 \mathrm{~cd} / \mathrm{m}^{2}$ ), Kremers and Scholl [16], who used a mean luminance of $66 \mathrm{~cd} / \mathrm{m}^{2}$, reported a large difference in slopes of the rod and cone ERG phase as a function of temporal frequency. The nearly parallel phase data for the isolated rod and cone stimuli in our study (see Fig. 2) probably are due to the low light level used in our study. As physiological recordings and psychophysical studies showed, rod and cone impulse response functions can be comparable at low mesopic light levels $[22,28]$. These results demonstrate that the four-primary method is a viable approach to separately record ERG responses from the rod and cone systems.

For the light level we used, the rod ERG amplitude was always larger than the cone ERG amplitude. From small-field psychophysical and retinal electro-physiological data, the cone contribution dominates at $20 \mathrm{Td}$ or higher light levels [14]. In full-field ERG measurements, the light range in which both rods and cones are active is even larger than estimated by small-field measurements, due to the summation of electrical responses from a very large number of rods. For instance, reliable rod ERGs have been recorded at a luminance as high as $66 \mathrm{~cd} / \mathrm{m}^{2}$, equal to a retinal illuminance of $3300 \mathrm{Td}$ with an $8-\mathrm{mm}$ pupil [16, 29]. This is higher than the rod-saturation level estimated by Aguilar and Stiles [30] in their classic 
psychophysical study. Historically, vision scientists have been aware of a rod contribution at high retinal illuminance level but have tried to minimize its influence [31]. When Stiles and Burch [32] measured large-field $10^{\circ}$ color-matching functions, they intentionally used extremely high-luminance stimuli to reduce rod intrusion. In this study, the measured phases were similar with those of the isolated rod stimuli and the combined stimuli (Fig. 2), also suggesting at the light level we used, the ERG signals were dominated by the rod contribution.

One significant advantage of the four-primary method is that it permits the separation of rod and cone ERGs under identical adaptation conditions. This allows for the assessment of rodcone interactions. Our results indicate that in normal observers, rod and cone ERG amplitudes were combined in a vector sum fashion. When we investigated the combination of rod and cone inputs in primate parasol ganglion cells using the four-primary method, we observed response saturation to the isolated rod or cone stimuli. Therefore, a vector model that included a saturation term was necessary to characterize ganglion cell responses [14]. Taking into account both the present results and those from ganglion cell recordings, it appears that rod and cone inputs are combined linearly prior to the saturation site. ERGs are believed to measure activities from the photoreceptor and bipolar cell levels [33]. Taken together, these results from current ERG measurements and primate ganglion cell recordings suggest that saturation occurs after the bipolar level in the retinal visual processing because primate ganglion cells showed saturated responses while photoreceptor or bipolar cells from ERG measurements did not.

ERG testing is invaluable in detecting photoreceptor function loss due to retinal diseases, particularly in those hereditary retinal diseases, such as RP [34, 35]. Affected rod and cone functions in many retinal eye diseases may change the pattern of rod-cone interactions and mesopic vision [36, 37]. It has been recognized that impaired mesopic vision is probably "the most sensitive and earliest sign" for many retinal diseases [38]. In a recent study [39], a significant association was found between mesopic critical fusion frequency obtained using a four-primary photostimulator and genetic risk of age-related maculopathy (ARM) in old subjects with normal fundoscopy, suggesting the importance of evaluating rod-cone interactions at mesopic light levels as a possible early sign of ARM. Rod-cone interactions assessed by mesopic ERGs using the four-primary method have the potential to be useful in the early detection of retinal diseases. Moreover, using the four-primary method to measure separate rod and cone ERGs at one mesopic light level could be more time efficient, particularly for testing patients, because it will take only a few minutes to test one frequency and light level. Most importantly, mesopic rod and cone ERG measurements can be safer and more comfortable by avoiding exposure to high-intensity light. It is suggested that prolonged light exposure for cone ERG measurement following the ISCEV standard protocol may have damaging effects on rods in some forms of RP [40]. Further, the standardized ERG techniques established by the ISCEV cannot be easily carried out with children because the protocols require long gaze fixation and attention spans [41]. Thus, by utilizing a light of moderate intensity, the mesopic ERGs should be well tolerated by children [42] and avoid any potential damages to photoreceptors from light adaptation [40].

One concern for applying the four-primary method in clinical ERG testing is the adequacy of receptor class isolation given interobserver variation in prereceptoral filtering. In this study, we used averaged correction factors from the five normal observers to account for pre-receptoral filtering in patients. Normal aging is known as a major factor affecting lens transmittance [43]. We calculated density function at the dominant wavelengths of the ColorDome LEDs (470, 524, 588, and 636) for a lens age between 25 and 65 years, using the equation provided by Pokorny et al. [44]. For each lens age, the rod and cone modulation contrasts were computed for the stimuli we used in the study with lens density considered. 
The results indicated that the error in photoreceptor silencing for the 65 year-old lens was very small. For the isolated rod stimuli ( $30 \%$ rod contrast; 0 cone contrast), the 65 -year-old lens had $29.0 \%$ rod contrast and $0.96 \%$ cone contrast; for the isolated cone stimuli ( 0 rod contrast; $30 \%$ cone contrast), the 65 year-old lens had $0.93 \%$ rod contrast and $30.96 \%$ cone contrast. These small isolation errors are negligible given that the ERG noise level was approximately $15 \%$ of the measured ERG amplitudes (Fig. 3). In other words, for clinical purposes, the observer calibration will not be critical.

\section{Acknowledgments}

This study was supported by National Eye Institute grant R01 EY019651 (D. C.), a seed grant from Illinois Society for Prevention of Blindness (D. C.) and the Institute for Translational Medicine Pilot and Collaborative

Translational and Clinical Studies Award (D. C.), National Eye Institute grant K08 EY019089-02 (M.A.G.), Hope for Vision (M. A. G.), and Foundation Fighting Blindness (M. A. G.). We are indebted to the observers and their families for their participation in the study. We thank Yolanda Lu for assisting in some of the data analyses.

\section{References}

1. Berson EL, Gouras P, Gunkel RD, Myrianthopoulos NC. Rod and cone responses in sex-linked retinitis pigmentosa. Arch Ophthalmol. 1969; 81:215-225. [PubMed: 5764685]

2. Berson EL, Gouras P, Gunkel RD. Rod responses in retinitis pigmentosa, dominantly inherited. Arch Ophthalmol. 1968; 80:58-67. [PubMed: 5660019]

3. Weymouth AE, Vingrys AJ. Rodent electroretinography: methods for extraction and interpretation of rod and cone responses. Prog Retin Eye Res. 2008; 27:1-44. [PubMed: 18042420]

4. Marmor MF, Fulton AB, Holder GE, Miyake Y, Brigell M, Bach M. ISCEV standard for full-field clinical electroretinography (2008 update). Doc Ophthalmol. 2009; 118(1):69-77. [PubMed: 19030905]

5. Abraham FA, Alpern M. Factors influencing threshold of the fundamental electrical response to sinusoidal excitation of human photoreceptors. J Physiol (Lond). 1984; 357:151-172. [PubMed: 6512688]

6. Shapiro AG, Pokorny J, Smith VC. Cone-rod receptor spaces, with illustrations that use CRT phosphor and light-emitting-diode spectra. J Opt Soc Am A. 1996; 13:2319-2328.

7. Pokorny J, Smithson H, Quinlan J. Photostimulator allowing independent control of rods and the three cone types. Vis Neurosci. 2004; 21:263-267. [PubMed: 15518198]

8. Ruddock KH. Evidence for macular pigmentation from colour matching data. Vis Res. 1963; 3:417429. [PubMed: 14168306]

9. Pokorny J, Smith VC. Effect of field size on red-green color mixture equations. J Opt Soc Am. 1976; 66:705-708. [PubMed: 978285]

10. Kremers J, Meierkord S. Rod-cone-interactions in deuteranopic observers: models and dynamics. Vis Res. 1999; 39:3372-3385. [PubMed: 10615502]

11. Buck, SL. Rod-cone interaction in human vision.. In: Chalupa, LM.; Werner, JS., editors. The visual neuroscience. Vol. 1. MIT Press; Cambridge: 2004. p. 863-878.

12. van den Berg TJTP, Spekreijse H. Interaction between rod and cone signals studied with temporal sine wave stimulation. J Opt Soc Am. 1977; 67:1210-1217. [PubMed: 903846]

13. MacLeod DI. Rods cancel cones in flicker. Nature. 1972; 235(5334):173-174. [PubMed: 4551230]

14. Cao D, Lee BB, Sun H. Combination of rod and cone inputs to in the parasol ganglion cells of the magnocellular pathway. J Vis. 2010; 10(11):4, 1-15. [PubMed: 20884499]

15. Miyake, Y. Electrodiagnosis of retinal diseases. Springer; Tokyo: 2006.

16. Kremers J, Scholl HPN. Rod-/L-cone and rod-/M-cone interactions in electroretinograms at different temporal frequencies. Vis Neurosci. 2001; 18:339-351. [PubMed: 11497411]

17. Estévez O, Spekreijse H. The "silent substitution" method in visual research. Vis Res. 1982; 22:681-691. [PubMed: 7112962]

18. Smith VC, Pokorny J. The design and use of a cone chromaticity space. Color Res Appl. 1996; 21:375-383.

Doc Ophthalmol. Author manuscript; available in PMC 2011 August 5. 
19. Puts MJH, Pokorny J, Quinlan J, Glennie L. Audiophile hardware in vision science; the soundcard as a digital to analog converter. J Neurosci Methods. 2005; 142(1):77-81. [PubMed: 15652619]

20. Cao D, Pokorny J, Smith VC. Matching rod percepts with cone stimuli. Vis Res. 2005; 45:21192128. [PubMed: 15845243]

21. Cao D, Pokorny J, Smith VC, Zele AJ. Rod contributions to color perception: linear with rod contrast. Vis Res. 2008; 48:2586-2592. [PubMed: 18561973]

22. Cao D, Zele AJ, Pokorny J. Linking impulse response functions to reaction time: rod and cone reaction time data and a computational model. Vis Res. 2007; 47:1060-1074. [PubMed: 17346763]

23. Cao D, Pokorny J. Rod and cone contrast gains derived from reaction time distribution modeling. J Vis. 2010; 10(2):11, 1-15. [PubMed: 20462312]

24. Sun H, Pokorny J, Smith VC. Rod-cone interactions assessed in inferred postreceptoral pathways. J Vis. 2001; 1:42-54. [PubMed: 12678613]

25. Le Grand, Y. Light, colour and vision. 2nd edn.. Chapman and Hall; London: 1968. p. 1-564.

26. Birch DG, Anderson JL, Fish GE. Yearly rates of rod and cone functional loss in retinitis pigmentosa and cone-rod dystrophy. Ophthalmol. 1999; 106(2):258-268.

27. Murray IJ, Kremers J, Parry NRA. L- and M-cone isolating ERGs: LED versus CRT stimulation. Vis Neurosci. 2008; 25(3):327-331. [PubMed: 18598403]

28. Schneeweis DM, Schnapf JL. Photovoltage of rods and cones in the macaque retina. Science. 1995; 268:1053-1056. [PubMed: 7754386]

29. Kremers J, Czop D, Link B. Rod and S-cone driven ERG signals at high retinal illuminances. Doc Ophthalmol. 2009; 118(3):205-216. [PubMed: 19101744]

30. Aguilar M, Stiles WS. Saturation of the rod mechanism of the retina at high levels of illumination. Opt Acta (Lond). 1954; 1:59-65.

31. Stiles WS, Wyszecki G. Rod Intrusion in large-field color matching. Acta Chromatica. 1973; 2(4): $155-163$.

32. Stiles WS, Burch JM. NPL colour-matching investigation: final report. Opt Acta (Lond). 1959; 6:1-26.

33. Miller RF, Dowling JE. Intracellular responses of the Muller (glial) cells of the mudpuppy retina: their relation to b-wave of the electroretinogram. J Neurophysiol. 1970; 33:323-341. [PubMed: 5439340]

34. Hartong DT, Berson EL, Dryja TP. Retinitis pigmentosa. Lancet. 2006; 368:1795-1809. [PubMed: 17113430]

35. Pagon RA. Retinitis pigmentosa. Surv Ophthalmol. 1988; 33:137-177. [PubMed: 3068820]

36. Arden GB, Hogg CR. Rod-cone interactions and analysis of retinal disease. Br J Ophthalmol. 1985; 69:404-415. [PubMed: 3873959]

37. Alexander KR, Fishman GA, Derlacki DJ. Mechanisms of rod-cone interaction: evidence from congenital stationary night blindness. Vis Res. 1988; 28:575-583. [PubMed: 3264093]

38. Petzold A, Plant GT. Clinical disorders affecting mesopic vision. Ophthalmic Physiol Opt. 2006; 26:326-341. [PubMed: 16684159]

39. Feigl B, Cao D, Morris CP, Zele AJ. Persons with age-related maculopathy risk genotypes and clinically normal eyes have reduced mesopic vision. Invest Ophthalmol Vis Sci. 2010; 52(2): 1145-1150. [PubMed: 20881291]

40. Cideciyan AV, Jacobson SG, Aleman TS, Gu D, Pearce-Kelling SE, Sumaroka A, Acland GM, Aguirre GD. In vivo dynamics of retinal injury and repair in the rhodopsin mutant dog model of human retinitis pigmentosa. Proc Natl Acad Sci USA. 2005; 102(14):5233-5238. [PubMed: 15784735]

41. Flores-Guevara R, Renault F, Ostre C, Richard P. Maturation of the electroretinogram in children: stability of the amplitude ratio a/b. Electroencephalogr Clin Neurophysiol. 1996; 100:422-427. [PubMed: 8893659]

42. Flores-Guevara R, Renault F, Loundon N, Marlin S, Pelosse B, Momtchilova M, Auzoux-Cheve M, Vermersch AI, Richard P. Usher syndrome type 1: early detection of electroretinographic changes. Eur J Pediatr Neurol. 2009; 13(6):505-507. 
43. Boettner EA, Wolter J. Transmission of the ocular media. Invest Ophthalmol Vis Sci. 1962; 1:776783.

44. Pokorny J, Smith VC, Lutze M. Aging of the human lens. Appl Opt. 1987; 26:1437-1440. [PubMed: 20454339] 

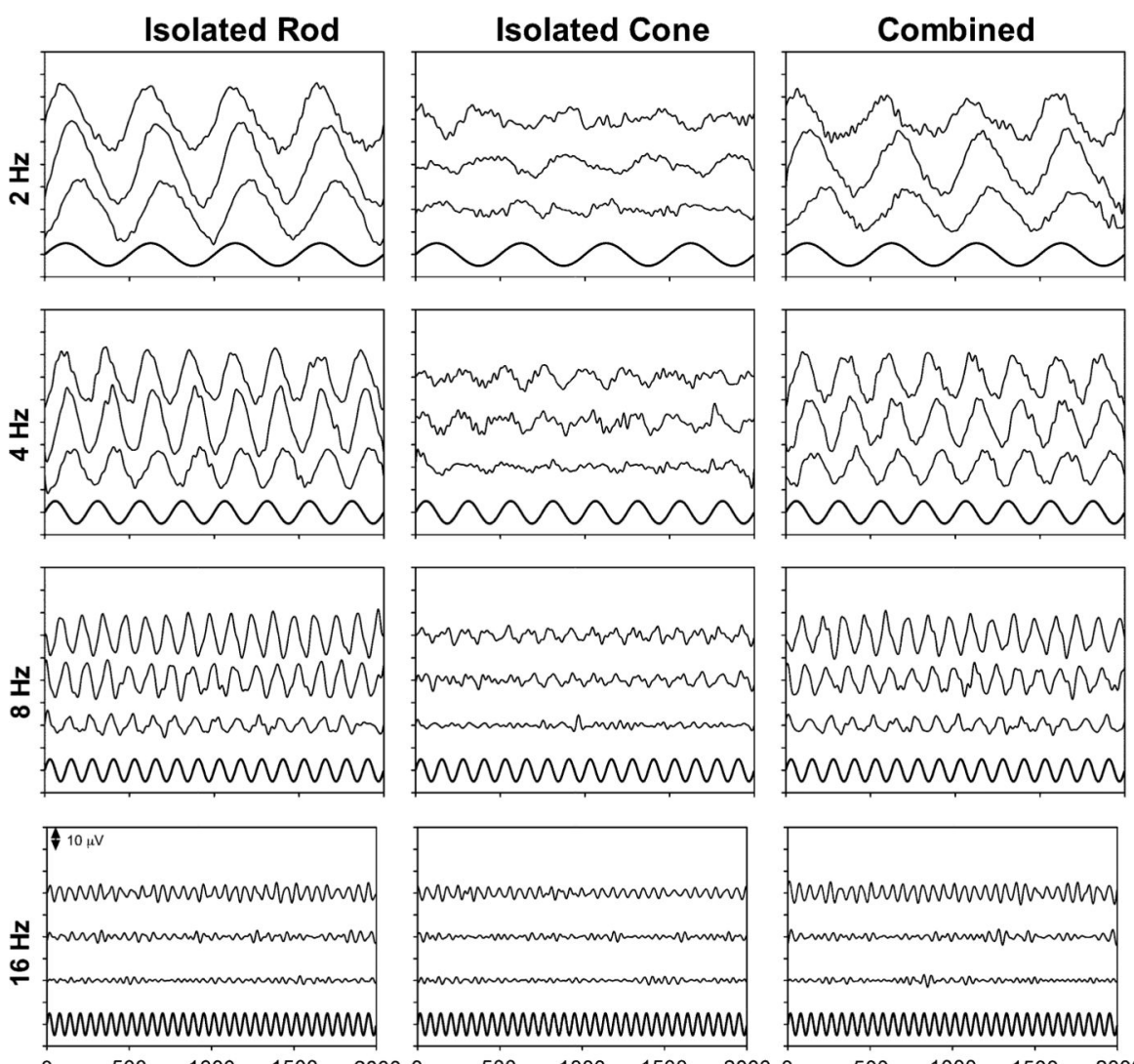

$0 \quad 500 \quad 1000 \quad 1500 \quad 2000$

$\begin{array}{llllllll}500 & 1000 \quad 1500 & 2000 & 0 & 500 & 1000 & 1500 & 2000 \\ & \text { Time (ms) }\end{array}$

Fig. 1.

The averaged ERG waveforms for one normal observer $(Y S)$ with the isolated rod stimuli (left column), isolated cone stimuli (middle column), or the combined stimuli (right column). The high-frequency components $(>30 \mathrm{~Hz})$ in each waveform were removed. Each panel shows the ERG waveforms with one stimulus type and one frequency at all three light levels (top trace for $1.26 \mathrm{~cd} / \mathrm{m}^{2}$, middle trace for $0.16 \mathrm{~cd} / \mathrm{m}^{2}$, and the bottom trace for $0.02 \mathrm{~cd} / \mathrm{m}^{2}$ ), with one unit in the y-axis for $10 \mu \mathrm{V}$. The sinusoidal stimulus is plotted in the bottom of each panel for reference purpose 

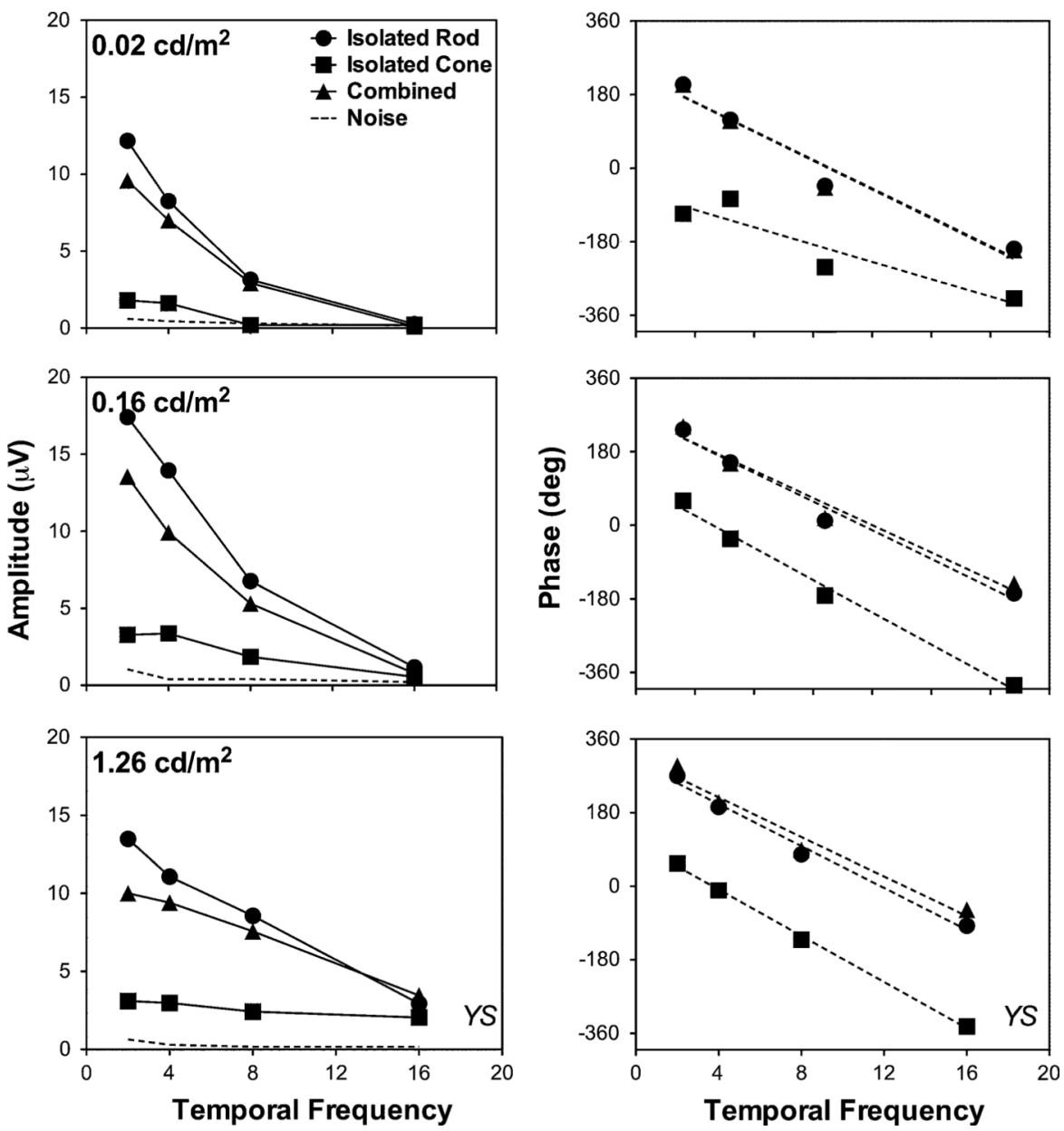

Fig. 2.

The ERG amplitudes (left column) and phases (right column) for the first harmonics extracted from the waveforms in Fig. 1. The dashed lines in the left column are the averaged noise amplitudes over three stimulus conditions at each light level, while the dashed lines in the right columns are linear regression fits 


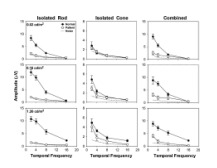

Fig. 3.

The averaged ERG amplitudes from all of the normal observers and the patients. The dashed lines are the averaged noise amplitudes from both the normal observers and patients. Note that the amplitudes for the isolated cone stimuli have different scales from those for the isolated rod or combined stimuli 


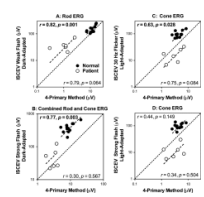

Fig. 4.

The correlation between the amplitudes of ERGs measured by the ISCEV standard protocol and those by the four-primary method in the normal observers and patients. Note that the amplitude for the ERGs measured by the four-primary method is the maximal amplitude across all temporal frequencies and light levels. The Pearson correlation and associated $P$ value are displayed on the top for normal observers and on the bottom for the patients in each panel. The data are plotted in log-log format with equal number of logarithmic scaling of the horizontal and vertical axes; therefore, the diagonal line (as indicated by the black dashed line) has a slope of 1.0. The other dashed lines are fits from simple linear regression without intercepts of the ERG amplitudes in normal observers or patients. The results demonstrate that the ERG amplitudes obtained from the four-primary method were largely proportional to those obtained from the ISCEV protocol. a ISCEV scotopic ERG b-wave amplitudes versus maximal rod ERG amplitude; b ISCEV Mixed rod and cone ERG b-wave amplitudes versus maximal combined rod and cone ERG amplitudes; c ISCEV 30-Hz flicker cone ERG amplitudes (peak-trough) versus cone ERG amplitudes; d ISCEV single bright flash ERG b-wave amplitudes versus cone ERG amplitudes 

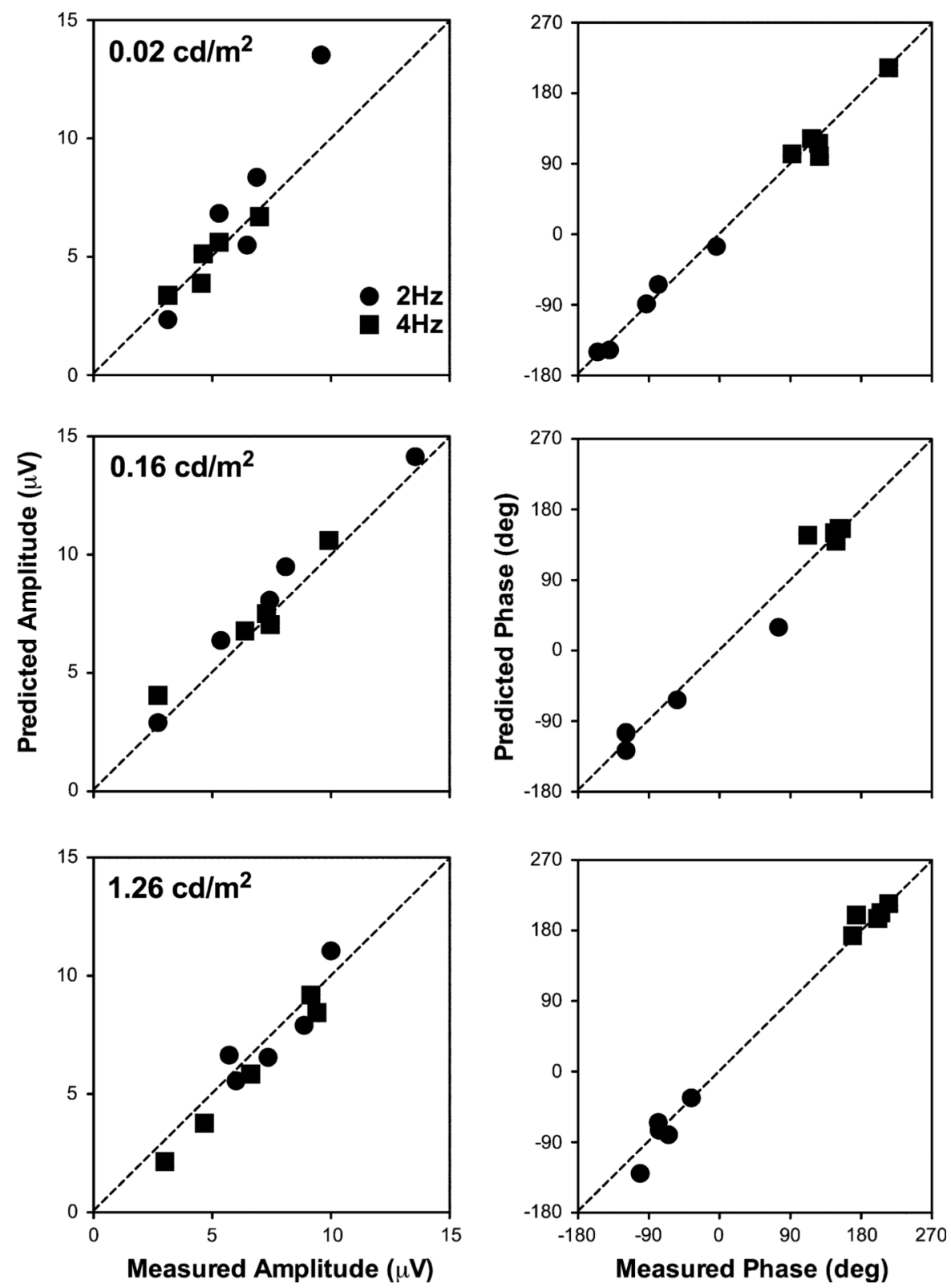

Fig. 5.

The predicted amplitudes and phases from the combined rod and cone stimuli based on the vector sum model versus the measured values in 5 normal observers tested for at least 3 sessions. In each panel, each of the 5 identical symbols represents one observer. The results at 8 and $16 \mathrm{~Hz}$ are not shown because of small response amplitudes at all light levels 